\title{
Structure of the active pharmaceutical ingredient bismuth subsalicylate
}

\author{
Erik Svensson Grape1, Victoria Rooth1', Mathias Nero1, Tom Willhammar ${ }^{1 *}$, A. Ken Inge ${ }^{1 *}$
}

\begin{abstract}
Structure determination of pharmaceutical compounds is invaluable for drug development but is challenging for materials that form as small crystals with defects. Bismuth subsalicylate (BSS), among the most commercially significant bismuth compounds, is an active ingredient in over-the-counter medications such as Pepto-Bismol (genericized trademark), used to treat dyspepsia and H. pylori infections. Despite its century-long history, the structure has remained unknown. Three-dimensional electron diffraction and hierarchical clustering analysis were applied on select data from ordered crystals, revealing a layered structure. In less ordered crystals high-resolution scanning transmission electron microscopy revealed various disorders, including variations in the stacking of layers. These modern electron crystallography techniques provide insight into differences in BSS crystals and the possibility to develop new analogs or polymorphs.
\end{abstract}

The physical, chemical and therapeutic properties of active pharmaceutical ingredients (APIs) are governed by their molecular structures and intermolecular interactions. Many APIs are crystalline substances, having periodic arrangements of their constituent molecules or ions. The specific arrangement of molecules in an API and their intermolecular interactions affect its stability and solubility, which in turn influences bioavailability, efficacy, and dosage. Therefore, determining the structures of pharmaceutical compounds is an integral part of drug formulation. Traditionally the method of choice for crystal structure determination has been single-crystal X-ray diffraction (SCXRD). However, the technique requires large specimens and is not readily applicable for sub-micrometer crystals. While structure determination of small crystallites can often be performed by powder X-ray diffraction (PXRD), the technique can at times struggle with complicated and disordered structures.

These reasons among others have previously prevented structure determination of the API bismuth subsalicylate (BSS), a compound made from $\mathrm{Bi}^{3+}$ cations and salicylic acid $\left(\mathrm{H}_{2} \mathrm{Sal}\right.$, Figure $\left.1 \mathrm{~A}\right)$. It is the API of popular over-thecounter medications such as Pepto-Bismol and commonly used to treat gastrointestinal disorders such as dyspepsia and diarrhea. Numerous studies have confirmed the efficacy of BSS as an antimicrobial, anti-inflammatory, and antacid agent (1-3). Recent studies have even demonstrated that bismuth compounds can even combat antibiotic resistance in bacteria (4).

BSS formulations were first developed in 1900 to treat Campylobacter infections, a major cause of infant deaths at the time (5). Since the discovery in the 1980s by Nobel laureates Barry Marshal and Robin Warren (6) of Helicobacter pylori, a bacterium harbored by $60 \%$ of the global population, bismuth compounds including BSS have been used to effectively treat peptic ulcer disease (7). In 1990, a report from Procter \& Gamble (P\&G) estimated that over 10 billion doses of Pepto-Bismol had been consumed and that it could be found in approximately $60 \%$ of U.S. households (5). In 2019 overall sales of more than 20 million units grossed over $\$ 100$ million in the U.S. alone, making it the most-sold stomach remedy in the country (8). Despite its century-long history and continuing widespread use, the structure of BSS has remained unknown and only a limited understanding has been established of its mechanisms of action.

Speculation on the formula and structure of BSS has been published in many chemical and pharmaceutical databases, websites, patents, textbooks, and articles, where BSS is often represented as a simple metal complex. Although efforts have been made to determine its crystal structure, obtaining sufficiently large specimens of BSS for SCXRD has not been possible - likely due to its poor solubility in water. Due to the difficulties in characterizing BSS, several model bismuth compounds have been developed through various approaches, including the synthesis of bismuth thiosalicylates (9), the incorporation of water or organic solvent molecules into the crystal structures (10-12), or by altering the Bi:Hsal stoichiometry (13), which is 1:1 for BSS.

To finally uncover the structure of BSS, we turned to two modern transmission electron microscopy (TEM) techniques: three-dimensional electron diffraction (3DED) (14) and high-resolution scanning transmission electron microscopy (STEM). 3DED techniques, such as CRED, ADT, fast-EDT, and MicroED, can be applied to obtain single-crystal diffraction data on crystallites that are even smaller than $200 \mathrm{~nm}$ for the determination of their average ordered structures. This has been facilitated through the development of methodology and hardware in recent years (15-20) and has allowed for faster and higher quality measurements for the structure determination of proteins $(16,18,21)$, inorganics (22), and organics $(23,24)$, including pharmaceuticals $(25,26)$ as well as various bismuth compounds $(27-$ 30). Concurrently, aberration-corrected STEM has evolved into an essential technique for atomic-scale structural investigations, particularly of local disorder. Recent development of STEM techniques such as integrated Differential Phase Contrast (iDPC) has allowed for studies on beam-sensitive specimens, including studies of organic molecules inside inorganic framework structures $(31,32)$.

${ }^{1}$ Department of Materials and Environmental Chemistry, Stockholm University, Stockholm, 10691, Sweden 
A<smiles>O=C(O)c1ccccc1O</smiles>
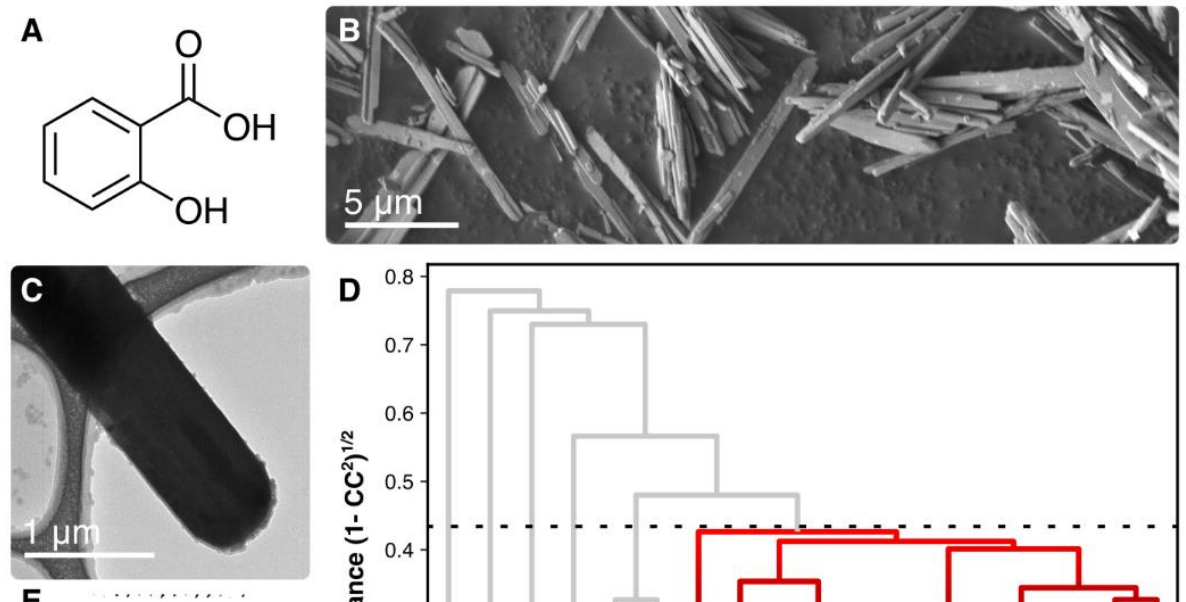

E

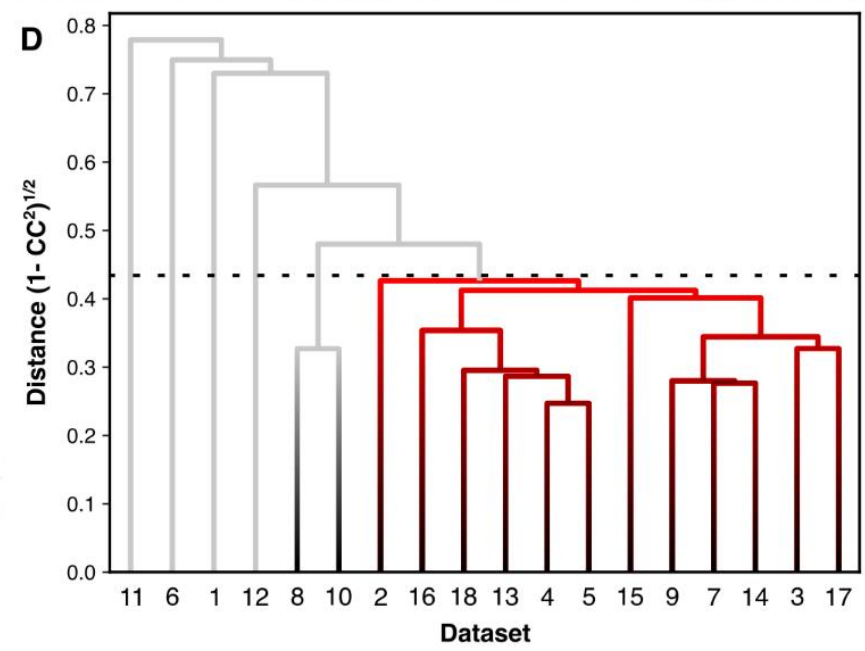

Fig. 1. Three-dimensional electron diffraction studies on BSS. (A) Molecular structure of salicylic acid $\left(\mathrm{H}_{2}\right.$ sal). (B) Scanning electron micrograph of bismuth subsalicylate from Sigma-Aldrich (BSS-SA). (C) One of 18 BSS-SA crystals used for the collection of 3DED data. (D) A dendrogram for the hierarchical clustering of the 18 individual 3DED datasets with the correlation coefficient cut-off shown as a dashed line. The red branch represents 12 highly correlated datasets with a distance below 0.44 , which were merged into a single combined 3DED dataset. (E) The reconstructed reciprocal space projection of a single 3DED dataset viewed along $\mathrm{C}^{*}$.

To identify appropriate BSS crystals for detailed investigations, five samples from different suppliers or formulations were screened by PXRD (Figure S1). All samples were crystalline and had characteristic PXRD patterns of BSS. Based on the quality of the PXRD patterns and commercial significance, our investigation narrowed its focus to two samples: BSS purchased from the chemical provider Sigma-Aldrich (BSS-SA) and BSS isolated from Pepto-Bismol original liquid formulation (BSS-PB).

Inspection of both BSS-SA and BSS-PB samples by scanning electron microscopy (SEM) and TEM imaging revealed crystals with a plank-shaped morphology that appeared homogeneous with no obvious indications of impurities (Figures 1B, S2, and S3). Despite efforts to further elucidate the structure by synchrotron SCXRD, the crystals proved too small and agglomerated. Structure determination by PXRD was not successful due to the preferred orientation of the crystals causing both over and underemphasized intensities, but also due to diffuse scattering and a rather complicated crystal structure, as disclosed by 3DED experiments.

3DED datasets were collected at $98 \mathrm{~K}$ on crystals from BSS-PB which had been centrifuged out of suspension and washed with water. The quality of datasets suffered from a variety of problems including inadequate data resolution, irregular peak shapes, twinning, and in some cases diffuse scattering (Figure S4, sample preparation, data collection, and data processing for all materials are described in the supplementary material). A few of the datasets could be indexed to a triclinic unit cell, however, no reasonable structure model could be obtained. Higher quality 3DED data were acquired from crystallites of BSS-SA (Figure S5). Datasets from 18 crystallites could be indexed with a triclinic unit cell $\left(a=8.35 \AA, b=12.17 \AA, c=18.09 \AA, \alpha=77.9^{\circ}, \beta=83.2^{\circ}, y=76.7^{\circ}\right)$. Structure solution was attempted on individual 3DED datasets but was unsuccessful. This was attributed to the low completeness of the individual datasets $(\leq 50 \%)$ caused by the low symmetry of the crystals and the limited tilt range intrinsic to the TEM.

To improve data completeness, hierarchical clustering analysis (HCA) was performed to merge datasets that were most similar in terms of measured intensities (33). A distance-metric based on the correlation coefficient (CC) of overlapping data was generated for all possible pairs of datasets. This resulted in two separate clusters with a CC of at least 0.90 , corresponding to a distance of 0.44 (Figure 1D). Structure solution was attempted with both clusters, yet only the large cluster of 12 individual datasets with an overall completeness of $84.6 \%$ proved successful (Figure 1D). Structure solution by direct methods resulted in a model with all non-hydrogen atoms appropriately located in the crystal structure with the space group $P-1$ (Table S1). 

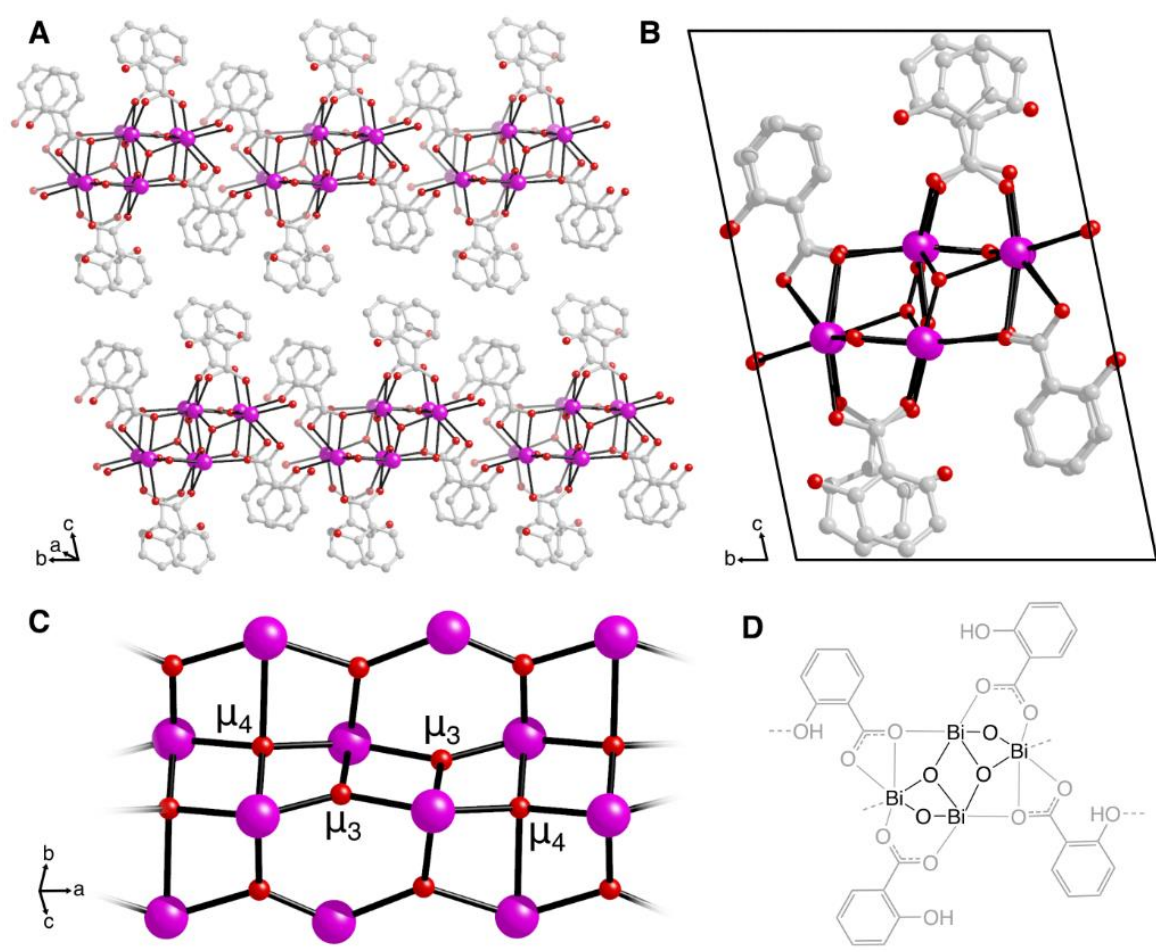

Fig. 2. The crystal structure of bismuth subsalicylate. (A) Stacking of the layers found in the BSS structure. Bi-O bonds are drawn as black lines. Hydrogen atoms have been omitted. (B) The unit cell of BSS viewed down the a-axis. (C) A section of the bismuthoxo rod forming the inorganic building unit which includes $\mathrm{O}^{2-}$ anions coordinated to alternatingly three and four $\mathrm{Bi}^{3+} \mathrm{cations} \mu_{3}$ and $\mu_{4}$ respectively. (D) A molecular sketch of a section of the BSS structure with bonds along the rod omitted for clarity.

As revealed by 3DED, BSS proved to be a coordination polymer with a layered structure (Figures 2A and S6). The crystal structure and its asymmetric unit of $\mathrm{Bi}_{4} \mathrm{O}_{4}(\mathrm{Hsal})_{4}$ is in accordance with the commonly presented empirical formula of $\mathrm{BiC}_{7} \mathrm{H}_{5} \mathrm{O}_{4}$, as well as with elemental and thermogravimetric analyses (Figure S7). In $\mathrm{BSS}, \mathrm{Bi}^{3+}$ cations are bridged by $\mathrm{O}^{2-}$ anions into bismuth-oxo rods which extend along the a-axis and form the inorganic building unit (IBU) of the structure (Figure $2 \mathrm{C}$ ). Along the center of the rods, $\mathrm{O}^{2-}$ anions bridge alternatingly three $\left(\mu_{3}\right)$ and four $\left(\mu_{4}\right) \mathrm{Bi}^{3+}$ cations. The IBU of BSS is nearly identical to one found in a previously reported bismuth-tricarboxylate coordination polymer (29). There are two types of salicylate anions ( $\mathrm{Hsal}^{-}$) in the BSS structure. One type of $\mathrm{Hsal}^{-}$coordinates via the carboxylate group only to $\mathrm{Bi}^{3+}$ cations of a single rod, while the phenol group does not coordinate to any $\mathrm{Bi}^{3+}$ cations. The other type also coordinates to $\mathrm{Bi}^{3+}$ cations through the carboxylate group; however, the phenol group coordinates to $\mathrm{Bi}^{3+}$ cations in adjacent rods, essentially linking the rods along the $b$-axis into centrosymmetric layers in the $a b$-plane. These layers stack along the $c$-axis and only weakly interact with one another via dispersion forces. As the unit cell is only one layer thick, IBUs in neighboring layers are oriented in the same direction in the ordered crystal. The protonation, as assigned in Figure 2D, results in a charge-balanced material with phenol groups still protonated and carboxylic acid groups deprotonated and coordinating to the $\mathrm{Bi}^{3+}$ cations. The carboxylate oxygen atoms form relatively shorter $\mathrm{Bi}-\mathrm{O}$ bonds $(2.6-2.8 \AA)$ compared to the phenol oxygen atoms $(2.8-2.9 \AA)$, which suggest deprotonation of the carboxylic acid of salicylic acid rather than the phenol group, which is also supported by IR spectra (Figure S8). Similar protonation assignment of the Hsal- ligands has been reported in structures such as $\left[\mathrm{Bi}_{4} \mathrm{O}_{2}(\mathrm{Hsal})_{8}\right] \cdot 2 \mathrm{MeCN} / \mathrm{MeNO}_{2}$ and $\left[\mathrm{Bi}(\mathrm{Hsal})_{3}\left(\mathrm{H}_{2} \mathrm{O}\right)\right](12,34)$.

Due to the poor quality of the 3DED data on BSS-PB crystals, PXRD data were instead utilized to investigate the structure of BSS-PB. Structure refinement of BSS-PB showed overall good agreement with the BSS structure obtained by 3DED on BSS-SA (Figure S9 and Table S2). However, diffuse scattering and asymmetric peak shapes in the PXRD pattern (Figure S10) suggested the presence of disorder in the BSS-PB samples.

To further validate the structure of BSS-PB and investigate structural disorder, aberration-corrected scanning transmission electron microscopy (STEM) imaging was applied. Imaging was performed using both annular dark-field (ADF) as well as integrated Differential Phase Contrast (iDPC) signals. The ADF contrast scales rapidly with the atomic number (proportional to $\mathrm{Z}^{1.5-2}$ ) thus highlighting heavier elements such as Bi. The iDPC contrast, on the other hand, scales linearly with atomic number, thereby emphasizing lighter elements when compared to ADF (35). 


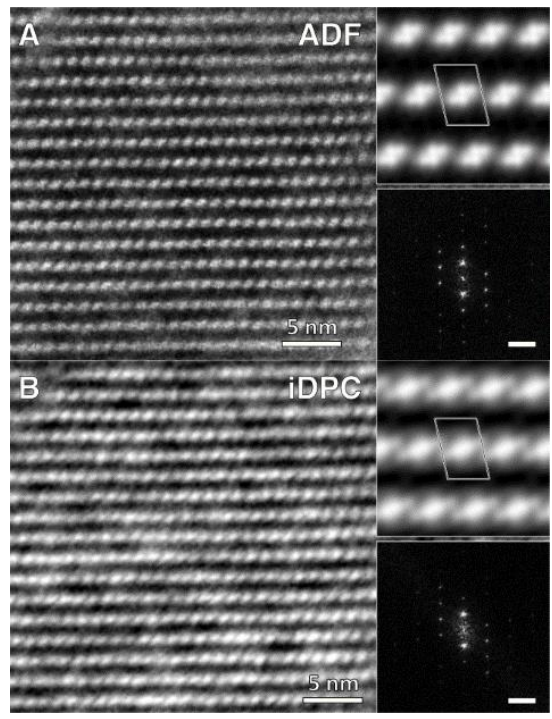

Fig 3. STEM images of a highly ordered section of a BSS-PB crystal along [100]. (A) Annular dark field (ADF) and (B) integrated Differential Phase Contrast (iDPC) scanning transmission electron microscopy (STEM) images. Lattice-averaged maps with p2 symmetry imposed and Fast Fourier transforms (scale bar is equal to $0.1 \AA^{-1}$ ) are shown as insets for each of the images.

Crystals of BSS-PB consisted of large ordered domains in projection consistent with the structure of BSS-SA determined by 3DED. Images along the [100] direction of BSS-PB revealed a similar orientation of the IBUs (Figure 3). The ADF contrast shows well-resolved projected positions of the $\mathrm{Bi}^{3+}$ ions of the IBU (Figure $3 \mathrm{~A}$ ), whereas the iDPC contrast, in addition, showed enhanced contrast in locations consistent with the positions of the salicylate anions, although not well resolved (Figure 3B).

However, upon inspection of other sections of the BSS-PB crystals, iDPC-STEM images revealed different types of disorder, particularly an inconsistency in the stacking of layers (Figures 4 and S11). It can be seen that the orientation of the layers vary, which can be caused by a two-fold rotation of the layers along the $b$-axis or perpendicular to the $a b$-plane. In some domains it was evident that the unit cell was doubled along the $c$-axis and the unit cell angle $a$ changed due to a periodic alternation of the layer orientation. In other domains the orientations of the layers appear to be random and the disorder is observed as diffuse scattering in the Fast Fourier transform (FFT) of the image, as shown in Figures 4D-4F. The fact that domains of disordered sequences are observed explains the occurrence of diffuse features in the FFT (Figure 4E), inconsistent peak shapes, and diffuse scattering in the PXRD pattern as well as the initial difficulties in obtaining a structure model.

As such, the material appears to have (1) ordered domains with a $c$-axis of $17 \AA$ with a single layer orientation (Figure $4 \mathrm{~A}$, area 1 , and $4 \mathrm{G}),(2)$ ordered domains with a doubled $c$-axis of $34 \AA$ and alternating layer orientation for adjacent layers (Figure $4 \mathrm{~A}$, area 3 , and $4 \mathrm{H}$ ), (3) domains of disordered stacking of the layers (Figures $4 \mathrm{~A}$, area 2, and $4 \mathrm{C}$ ), as well as (4) defects where the orientation changes within an individual layer (Figure 4B).

Other forms of defects were also observed, such as fringing of the (010) facets, as well as bending, buckling, and separation of the layers in some crystals (Figure S12). While this perturbation could be attributed to embedding the BSS crystals in the resin during sample preparation, their presence is likely a consequence of the weak interactions between the individual layers of the structure. However, it should be noted that most of the crystals and domains exhibited periodicity with an ordered $17 \AA$ c-axis consistent with the structure obtained from the clustered 3DED data.

Elucidation of the structure of commercial BSS provides a major step towards understanding the properties of one of the most commercially significant bismuth compounds. The fact that BSS is practically insoluble in water and the hydrophobic properties of the powder can be partly attributed to the continuous structure of the coordination polymer, where the nonpolar section of the salicylate anions form the outer surfaces of the layers, while all polar components, such as the phenol, carboxylate, $\mu_{3}-\mathrm{O}^{2-}, \mu_{4}-\mathrm{O}^{2-}$ and $\mathrm{Bi}^{3+}$, are contained within the layers. In addition to its high stability in water, BSS also demonstrated decent stability in aqueous solutions of HCl (Figure S13). No changes were observed in the PXRD patterns of BSS treated at a pH of 3 or higher. At a $\mathrm{pH}$ of 2, a small proportion of BSS converted into bismuth oxychloride, and was fully converted at a pH of 1. Considering this, interactions between the hydrophobic exterior of the BSS crystals and the gastric lining could to some extent govern the pharmacodynamics of this long-used formulation (36). 


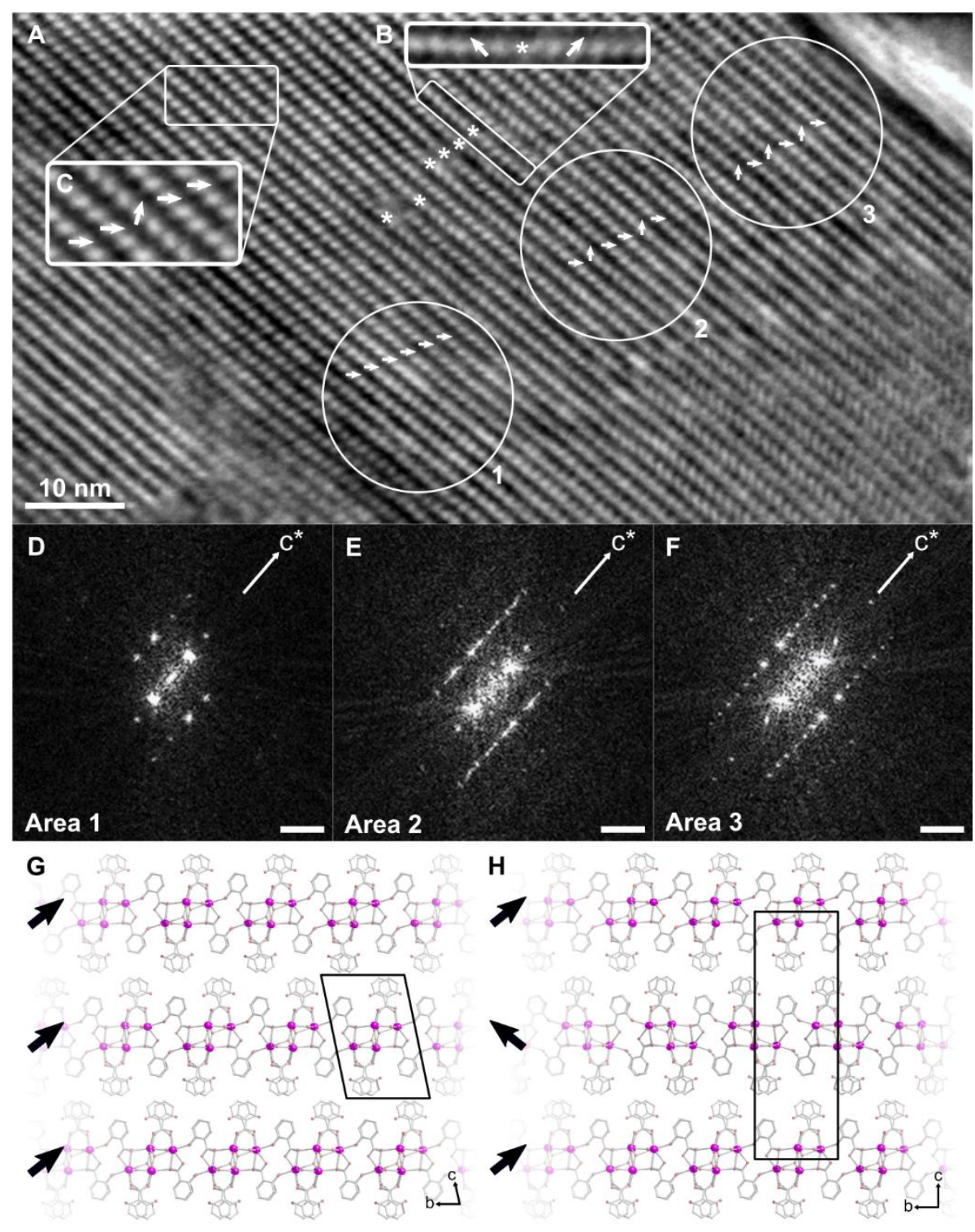

Fig 4. iDPC-STEM image of various forms of disorder in BSS-PB. (A, B, C) Domains of disorder are found in BSS while imaged along the [100] direction, showing alternation of the layer orientation both between and within the layers. Arrows indicate the orientation of the layers. Stars $\left(^{*}\right)$ indicate switching of the orientation within a single layer. (D) Domains with a $17 \AA c$-axis are found as evidenced by the Fast Fourier transform (FFT), showing a single layer orientation. (E) Domains of disordered stacking are also observed as diffuse streaking perpendicular to the layer. (F) Domains showing a doubled $34 \AA c$-axis, arising from an alternating orientation of the individual layers (scale bar in $D, E$, and $F$ corresponds to $0.1 \AA^{-1}$ ). (G) The structure of BSS as determined from 3DED data with all similar orientations of the layers and $(\mathrm{H})$ a structure with alternating layer orientation consistent with the doubled c-axis.

The various forms of analysis including PXRD, 3DED, and STEM imaging indicated that the two investigated samples, BSS-PB and BSS-SA, were built from the same layers but that the samples differed in terms of the degree of crystallinity and disorder in their structures. As such it is expected that the stacking of the layers is a consequence of the synthesis conditions utilized by various producers. Understanding the differences in local ordering of BSS opens opportunities to develop new analogs containing unique stacking sequences or higher degrees of exfoliation which may influence the properties and the efficacy of the API. 


\section{References}

1. G. G. Briand, N. Burford, Bismuth Compounds and Preparations with Biological or Medicinal Relevance. Chem. Rev. 99, 2601-2658 (1999).

2. P. J. Sadler, H. Li, H. Sun, Coordination chemistry of metals in medicine: target sites for bismuth. Coord. Chem. Rev. 185186, 689-709 (1999).

3. D. Y. Graham, S. Y. Lee, How to effectively use bismuth quadruple therapy: the good, the bad, and the ugly. Gastroenterol. Clin. North Am. 4, 537-563 (2015).

4. R. Wang, T. P. Lai, P. Gao, H. Zhang, P. L. Ho, P. C. Y. Woo, G. Ma, R. Y. T. Kao, H. Li, H. Sun, Bismuth antimicrobial drugs serve as broad-spectrum metallo- $\beta$-lactamase inhibitors. Nat. Commun. 9 (2018), doi:10.1038/s41467-018-02828-6.

5. D. W. Bierer, Bismuth Subsalicylate: History, Chemistry, and Safety Author ( s ): Douglas Ws . Bierer Source: Reviews of Infectious Diseases, Vol . 12, Supplement 1. Pathophysiology of Gastrointestinal Infections: The Role of Bismuth Subsalicylate ( Jan . - Feb. Oxford Univ. Press. 12 (1990) (available at http://www.jstor.org/stable/4455445).

6. B. Marshall, R. Warren, Unidentified curved bacilli on gastric epithelium in active chronic gastritis. Lancet. 321, 1273-1275 (1983).

7. J. M. Liou, M. S. Wu, J. T. Lin, Treatment of Helicobacter pylori infection: Where are we now? J. Gastroenterol. Hepatol. 31, 1918-1926 (2016).

8. S. Mendelson, Ed., Drug Store News, NACDS Total Store Expo, 2019. Drug Store News (2019), p. 38.

9. N. Burford, M. D. Eelman, T. S. Cameron, Comprehensive characterisation of bismuth thiosalicylate complexes: Models for bismuth subsalicylate. Chem. Commun. 13, 1402-1403 (2002)

10. J. H. Thurston, E. M. Marlier, K. H. Whitmire, Towards a molecular model for bismuth(III) subsalicylate. Synthesis and solidstate structure of $[\mathrm{Bi}(\mathrm{Hsal}) 3$ (bipy) $(\mathrm{C} 7 \mathrm{H} 8] 2$ and $[\mathrm{Bi}(\mathrm{Hsal})($ sal) $(1,10-$ phenanthroline)(C7H8]2. Chem. Commun. 23, 23842835 (2002).

11. P. C. Andrews, G. B. Deacon, C. M. Forsyth, P. C. Junk, I. Kumar, M. Maguire, Towards a structural understanding of the anti-ulcer and anti-gastritis drug bismuth subsalicylate. Angew. Chemie - Int. Ed. 45, 5638-5642 (2006).

12. T. D. Boyd, I. Kumar, E. E. Wagner, K. H. Whitmire, Synthesis and structural studies of the simplest bismuth(III) oxosalicylate complex: [Bi4( $\mu 3-0) 2(\mathrm{HO}-2-\mathrm{C} 6 \mathrm{H} 4 \mathrm{CO} 2) 8] \cdot 2 \mathrm{Solv}$ (Solv = MeCN or MeNO2). Chem. Commun. 50, 3556-3559 (2014).

13. V. André, A. Hardeman, I. Halasz, R. S. Stein, G. J. Jackson, D. G. Reid, M. J. Duer, C. Curfs, M. T. Duarte, T. Friščić, Mechanosynthesis of the metallodrug bismuth subsalicylate from Bi2O3 and structure of bismuth salicylate without auxiliary organic ligands. Angew. Chemie - Int. Ed. 50, 7858-7861 (2011).

14. M. Gemmi, E. Mugnaioli, T. E. Gorelik, U. Kolb, L. Palatinus, P. Boullay, S. Hovmöller, J. P. Abrahams, 3D electron diffraction: The nanocrystallography revolution. ACS Cent. Sci. 5, 1315-1329 (2019).

15. U. Kolb, T. Gorelik, C. Kübel, M. T. Otten, D. Hubert, Towards automated diffraction tomography: Part I-Data acquisition. Ultramicroscopy. 107, 507-513 (2007).

16. D. Shi, B. L. Nannenga, M. G. Iadanza, T. Gonen, Three-dimensional electron crystallography of protein microcrystals. Elife. 2, e01345 (2013).

17. I. Nederlof, E. van Genderen, Y.-W. Li, J. P. Abrahams, A Medipix quantum area detector allows rotation electron diffraction data collection from submicrometre three-dimensional protein crystals. Acta Crystallogr. Sect. D Biol. Crystallogr. 69, 1223-1230 (2013).

18. B. L. Nannenga, D. Shi, A. G. W. Leslie, T. Gonen, High-resolution structure determination by continuous-rotation data collection in MicroED. Nat. Methods. 11, 927 (2014).

19. M. Gemmi, M. G. I. La Placa, A. S. Galanis, E. F. Rauch, S. Nicolopoulos, Fast electron diffraction tomography. J. Appl. Crystallogr. 48, 718-727 (2015).

20. M. O. Cichocka, J. Ångström, B. Wang, X. Zou, S. Smeets, High-throughput continuous rotation electron diffraction data acquisition via software automation . J. Appl. Crystallogr. 51, 1652-1661 (2018).

21. H. Xu, H. Lebrette, M. T. B. Clabbers, J. Zhao, J. J. Griese, X. Zou, M. Högbom, Solving a new R2lox protein structure by microcrystal electron diffraction. Sci. Adv. 5, 1-7 (2019).

22. B. Lu, T. Willhammar, B.-B. Sun, N. Hedin, J. D. Gale, D. Gebauer, Introducing the crystalline phase of dicalcium phosphate monohydrate. Nat. Commun. 11, 1-8 (2020).

23. C. G. Jones, M. W. Martynowycz, J. Hattne, T. J. Fulton, B. M. Stoltz, J. A. Rodriguez, H. M. Nelson, T. Gonen, The CryoEM Method MicroED as a Powerful Tool for Small Molecule Structure Determination. ACS Cent. Sci. 4, 1587-1592 (2018).

24. C. Gao, J. Li, S. Yin, G. Lin, T. Ma, Y. Meng, J. Sun, C. Wang, Isostructural Three-Dimensional Covalent Organic Frameworks. Angew. Chemie - Int. Ed. 58, 9770-9775 (2019).

25. T. Gruene, J. T. C. Wennmacher, C. Zaubitzer, J. J. Holstein, J. Heidler, A. Fecteau-Lefebvre, S. De Carlo, E. Müller, K. N. Goldie, I. Regeni, T. Li, G. Santiso-Quinones, G. Steinfeld, S. Handschin, E. van Genderen, J. A. van Bokhoven, G. H. Clever, R. Pantelic, Rapid Structure Determination of Microcrystalline Molecular Compounds Using Electron Diffraction. 
Angew. Chemie - Int. Ed. 57, 16313-16317 (2018).

26. P. Brázda, L. Palatinus, M. Babor, Electron diffraction determines molecular absolute configuration in a pharmaceutical nanocrystal. Science (80-. ). 364, 667-669 (2019).

27. M. Feyand, E. Mugnaioli, F. Vermoortele, B. Bueken, J. M. Dieterich, T. Reimer, U. Kolb, D. De Vos, N. Stock, Automated diffraction tomography for the structure elucidation of twinned, sub-micrometer crystals of a highly porous, catalytically active bismuth metal-organic framework. Angew. Chemie - Int. Ed. 51, 10373-10376 (2012).

28. Y. Wang, S. Takki, O. Cheung, H. Xu, W. Wan, L. Öhrström, A. K. Inge, Elucidation of the elusive structure and formula of the active pharmaceutical ingredient bismuth subgallate by continuous rotation electron diffraction. Chem. Commun. 53, 7018-7021 (2017)

29. E. Svensson Grape, H. Xu, O. Cheung, M. Calmels, J. Zhao, C. Dejoie, D. M. Proserpio, X. Zou, A. Ken Inge, Breathing Metal-Organic Framework Based on Flexible Inorganic Building Units. Cryst. Growth Des. 20, 320-329 (2019).

30. E. S. Grape, J. G. Flores, T. Hidalgo, E. Martínez-Ahumada, A. Gutiérrez-Alejandre, A. Hautier, D. R. Williams, M. O’Keeffe, L. Öhrström, T. Willhammar, P. Horcajada, I. A. Ibarra, A. K. Inge, A Robust and Biocompatible Bismuth Ellagate MOF Synthesized Under Green Ambient Conditions. J. Am. Chem. Soc. 142, 16795-16804 (2020).

31. B. Shen, X. Chen, H. Wang, H. Xiong, E. G. T. Bosch, I. Lazić, D. Cai, W. Qian, S. Jin, X. Liu, Y. Han, F. Wei, A singlemolecule van der Waals compass. Nature. 592, 541-544 (2021).

32. J. Cho, Y. Yun, H. Xu, J. Sun, A. W. Burton, K. G. Strohmaier, G. Terefenko, H. Vroman, M. Afeworki, G. Cao, H. Wang, X. Zou, T. Willhammar, EMM-25: The Structure of Two-Dimensional $11 \times 10$ Medium-Pore Borosilicate Zeolite Unraveled Using 3D Electron Diffraction. Chem. Mater. 33, 4146-4153 (2021).

33. B. Wang, X. Zou, S. Smeets, Automated serial rotation electron diffraction combined with cluster analysis: An efficient multi-crystal workflow for structure determination. IUCrJ. 6, 854-867 (2019).

34. I. Kumar, P. Andrews, K. H. Whitmire, The unexpected isolation of bismuth tris(carboxylate) hydrates: Syntheses and structures of $[\mathrm{Bi}(\mathrm{Hsal}) 3(\mathrm{H} 2 \mathrm{O})]$ and $[\mathrm{Bi}($ hanth) $3(\mathrm{H} 2 \mathrm{O})](\mathrm{H} 2 \mathrm{sal}=2-\mathrm{OH}-\mathrm{C} 6 \mathrm{H} 4 \mathrm{CO} 2 \mathrm{H}$, Hanth $=2-\mathrm{NH} 2-\mathrm{C} 6 \mathrm{H} 4 \mathrm{CO} 2 \mathrm{H})$. Eur. J. Inorg. Chem. 2015, 605-608 (2015).

35. I. Lazić, E. G. T. Bosch, S. Lazar, Phase contrast STEM for thin samples: Integrated differential phase contrast. Ultramicroscopy. 160, 265-280 (2016).

36. P. M. Goggin, J. M. Marrero, R. T. Spychal, P. A. Jackson, C. M. Corbishley, T. C. Northfield, Surface hydrophobicity of gastric mucosa in Helicobacter pylori infection: Effect of clearance and eradication. Gastroenterology. 103, 1486-1490 (1992). 


\section{Acknowledgements}

Use of the Advanced Photon Source at Argonne National Laboratory was supported by the U.S. Department of Energy, Office of Science, Office of Basic Energy Sciences, under Contract No. DE-AC02-06CH11357. A.K.I. would like to thank Prof. Tomislav Friščić for inspirational correspondence, John Inge for proof-reading and improving the manuscript, as well as Dr. Catherine Dejoie and Dr. William Shepard for data collection. Use of the Advanced Photon Source at Argonne National Laboratory was supported by the U. S. Department of Energy, Office of Science, Office of Basic Energy Sciences, under Contract No. DE-AC02-06CH11357.

\section{Funding}

E.S.G. and A.K.I. are supported by the Swedish Foundation for Strategic Research (SSF). A.K.I. is also supported by the Knut and Alice Wallenberg Foundation (KAW 2016.0072). T.W acknowledges support from the Swedish Research Council (VR, 2019-05465).

\section{Author Contributions}

E.S.G. prepared the samples, performed and analyzed 3DED, HCA, PXRD, SEM, TGA and FT-IR data as well as structure solution and refinement. V.R. prepared samples and helped perform PXRD and SEM. M.N. prepared and sectioned samples for STEM imaging. T.W. performed STEM analysis and managed TEM experiments. A.K.I. managed the project. E.S.G., T.W. and A.K.I performed the final structure elucidation and wrote the manuscript. All authors provided feedback and discussed the manuscript.

\section{Competing Interests}

Authors declare no competing interests.

\section{Data and Material Availability}

All data is available in the main text or the supplementary materials. CCDC 2095448 contains the supplementary crystallographic data for this paper. These data can be obtained free of charge via www.ccdc.cam.ac.uk/data_request/cif, or by emailing data_request@ccdc.cam.ac.uk, or by contacting The Cambridge Crystallographic Data Centre, 12 Union Road, Cambridge CB2 1EZ, U.K.; fax: + 441223336033 .

\section{Supplementary Material}

Materials and Methods.

Figure S1-S13.

Table S1-S2. 